\title{
An Analysis of the International and Domestic Health Hazards Posed by the 2014 West African Ebola Virus Disease Outbreak
}

\author{
Andrew Hickey, Sarah Cheeseman Barthel, Tyann Blessington*, Yandace K. Brown, Diana \\ Y. Wong, William N. Albrecht, Mark Freese, Teresa Quitugua
}

National Biosurveillance Integration Center, Washington, District of Columbia, USA

\section{Objective}

To categorize and assess the international and domestic health impacts of the 2014 West African Ebola Virus Disease outbreak

\section{Introduction}

An epidemic of ebolavirus in West Africa, which was first identified in March 2014, is now the largest Ebola Virus Disease (EVD) outbreak on record. On 8 August 2014, the WHO declared the Ebola outbreak in West Africa a Public Health Emergency of International Concern (PHEIC). As of 4 September 2014, Guinea, Liberia, Sierra Leone, and Nigeria have reported 3,707 cases (2,106 confirmed, 1,003 probable, and 598 suspected) of EVD with 1,848 deaths (50\% case fatality) to the World Health Organization (WHO). Five U.S. citizens have contracted the viral disease - one LiberianAmerican and four medical-aid workers working in Ebola-afflicted countries.

\section{Methods}

In collaboration with multiple partners, the National Biosurveillance Integration Center (NBIC) provided an integrated analysis of the international and domestic health impacts of the West African EVD outbreak. NBIC began reporting on EVD on 24 March 2014, after a Guinean outbreak of hemorrhagic fever was confirmed to be ebolavirus, through briefs to Department of Homeland Security (DHS) components and OHA leadership and through timely situational reports to Federal, state, and local partners. Situational reports were distributed through multiple DHS web-based portals and were also distributed to key personnel in DHS leadership. Informational exchange agreements were developed to share NBIC reports with Homeland Security Advisory Council staff and, at the request of the US Department of Transportation, Transportation Canada. NBIC analysts worked with the Armed Forces Health Surveillance Center (AFHSC) to identify and monitored the health status of suspected EVD cases having a history of travel from or within the West African region and provided daily updates to OHA senior leadership and the Department of Transportation. NBIC analysts evaluated air-travel and migration patterns of West African residents in collaboration with Customs and Border Protection (CBP), Office of Intelligence and Investigative Liaison (OIIL). An NBIC analyst also deployed to the Health and Human Services (HHS) Secretary's Operations Center (SOC) for the initiation and the timely transfer of information regarding this epidemic. NBIC requested and shared information with other US Government biosurveillance analysts through the Wildfire portal governed by the Biosurveillance Indications and Warning Analytic Community (BIWAC), and provided or facilitated responses to requests for information (RFIs) from interagency agencies and groups. NBIC representatives also presented and participated in various Ebola working groups and inter-agency collaborative groups within the Federal government. Additionally, NBIC analysts collaborated with partners in an interagency Ebola modeling coordination group tasked to assess the current disease status and predicts future trends. These collaborative efforts and extensive information exchanges facilitated the development of guidance documents on screening procedures at airports and the assessment of the translocation rate and spread of the virus from Africa to the U.S. for the DHS Secretary and component agencies leaders.

\section{Results}

The current West Africa outbreak is believed to have originated in rural areas but subsequently spread to more urban centers. Many health centers in West Africa are reported to be underequipped and overwhelmed. Limited resources, high patient-provider ratio, poor training of healthcare workers, and a strong mistrust and apprehension of the local population towards health-guidance measures administered by the government create a challenge to maintain proper clinical infection control techniques. In general, the health security capacity to mitigate biological threats is significantly greater in the U.S. when compared to many regions in West Africa. Many of the West Africa EVD-afflicted nations are resource-poor and already coping with major health challenges.

\section{Conclusions}

The West African epidemic will only be quelled through widespread adherence of public health initiatives promoting barriernursing techniques, health education, and the rapid identification of cases. In contrast to the capabilities in West Africa, the U.S. health system is well-equipped to treat and contain trans located cases. The ongoing EVD outbreak in West Africa is unlikely to affect public health in the U.S. significantly.

\section{Keywords}

Ebola, West Africa, Outbreak.

*Tyann Blessington

E-mail: Tyann.Blessington@hq.dhs.gov 